\title{
A jellium model of a catalyst particle in carbon nanotube growth
}

\author{
Vasilii I. Artyukhov, Mingjie Liu, Evgeni S. Penev, and Boris I. Yakobson a) \\ Department of Materials Science and NanoEngineering, Rice University, Houston, Texas 77005, USA
}

(Received 4 April 2017; accepted 6 June 2017; published online 23 June 2017)

\begin{abstract}
We show how a jellium model can represent a catalyst particle within the density-functional theory based approaches to the growth mechanism of carbon nanotubes (CNTs). The advantage of jellium is an abridged, less computationally taxing description of the multi-atom metal particle, while at the same time in avoiding the uncertainty of selecting a particular atomic geometry of either a solid or ever-changing liquid catalyst particle. A careful choice of jellium sphere size and its electron density as a descriptive parameter allows one to calculate the CNT-metal interface energies close to explicit full atomistic models. Further, we show that using jellium permits computing and comparing the formation of topological defects (sole pentagons or heptagons, the culprits of growth termination) as well as pentagon-heptagon pairs 517 (known as chirality-switching dislocation). Published by AIP Publishing. [http://dx.doi.org/10.1063/1.4986949]
\end{abstract}

\section{INTRODUCTION}

Unique electronic and mechanical properties of carbon nanotubes (CNTs) promise a broad spectrum of applications. It would be fair to say that the research efforts have brought this material to the stage of upward slope of sustainable development in the "hype cycle." "For electronics, even presently high price of the CNT $(\sim 1 \mathrm{k} \$ / \mathrm{g})$ is not considered as an obstacle. ${ }^{2}$ What still remains a challenge is the practical control of the single-walled CNT symmetry type, the chirality $(n, m)$.

In understanding the origin of CNT chirality and the overall process of synthesis via catalytic chemical vapor deposition (CVD), computational modeling has provided insight into both thermodynamics ${ }^{3,4}$ and growth kinetics. ${ }^{5}$ By combining both factors, we have recently developed a theory of nanotube chirality ${ }^{6}$ that could not only explain the unusual near-armchair $(n, n-1)$ preference in numerous CVD experiments, ${ }^{7-10}$ but also suggests a possible rationale for the very recent reports of practically single-chirality samples. ${ }^{11,12}$ Indeed, this theory underscores the role of the catalyst/CNT interface, essentially a contact between the CNT edge and $\sim(n+m)$ atoms on the catalyst particle surface, with specifics determined by the state of the catalysts: solid/rigid vs liquid/compliant, as the stage where the chirality of a nascent CNT is established. Detailed description of the processes at the interface has been routinely fully atomistic, for the whole catalyst/CNT system, with interactions modeled at different levels of theory, from empirical force fields, ${ }^{13,14}$ to tight binding, ${ }^{15,16}$ and to first-principles density-functional theory (DFT) ${ }^{17}$ Since the system size is the major bottleneck in these schemes, in most studies, and especially those using DFT, the catalyst particle is typically a small metal cluster, $\sim 50$ atoms or less. Even in this size range, mainly static, total-energy DFT calculations are feasible, whereas bridging

a)Electronic mail: biy @ rice.edu any time scales of relevance to the CNT growth is unaffordable within $a b$ initio molecular dynamics. The major computational burden is associated with the catalyst, although rather large portion of it is not involved into CNT growth process. Moreover, at the atomistic level, the very choice of exact positions for each constituent atom of the catalysts is inevitably uncertain, especially for the ever-changing liquid particle.

As a remedy, here we propose a jellium sphere ${ }^{18,19}$ (“Je") as a catalyst model that allows for considerable reduction of configurational space by replacing the ionic cores with an uniform positive charge density, and thereby reducing computational burden, preserving at the same time sufficient accuracy in describing the catalyst/CNT interface within DFT. By construction, such a Je model corresponds to an idealized, rigid catalyst-sphere of radius $R$. We first explore the basic energetics of C-Je interactions and then assess the ability to capture the essential physics at the $\mathrm{CNT} / \mathrm{Je}$ interface.

\section{RESULTS AND DISCUSSION}

The "jellium" is essentially a single-parameter model, where its properties are defined by the jellium density, usually specified via the Wigner-Seitz radius $r_{s}$, so that the constant positive background density is $\rho_{+}=\left(4 \pi r_{s}^{3} / 3\right)^{-1}$. The radius of a Je sphere containing $N$ electrons is then $R=r_{s} N^{1 / 3}$, and one has the freedom to choose either $N$ or $r_{s}$ as the model descriptor-parameter.

Since simple carbon species ( $\mathrm{C}$ monomer, $\mathrm{C}_{2}$ dimer) are the initial product of decomposing a common feedstock precursor in CVD, we first quantify their binding to Je. DFT calculations, using the QUANTUM ESPRESSO package, ${ }^{21}$ are performed within the local density approximation for two Je spheres with $R=4.5 \AA$ and $4.1 \AA$. The setup employs a planewaves basis set of 30 Ry kinetic energy cutoff. The Je sphere is represented as a pseudo-atom with $Z=N$ valence electrons 

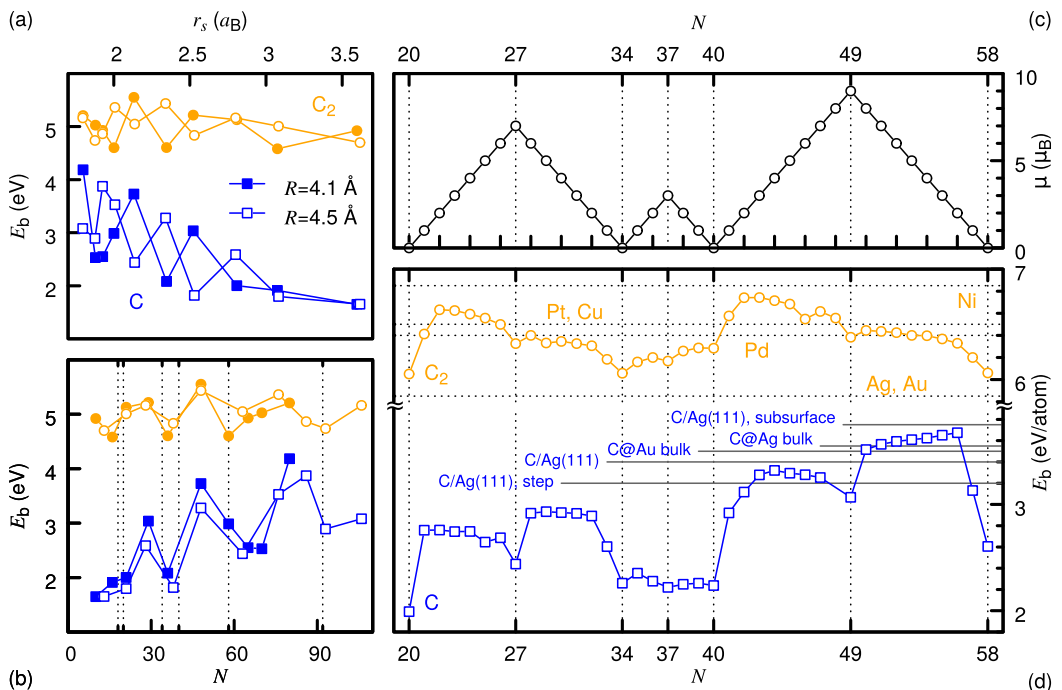

FIG. 1. (a) Binding energy $E_{\mathrm{b}}$ of carbon monomer C and dimer $\mathrm{C}_{2}$ on Je spheres with different radii $R$ as a function of Wigner-Seitz radius $r_{s}$ and (b) as a function of number of electrons $N$; grid lines indicate the magic numbers 18, 20,34, 40, 58, 92. (c) Magnetic moment of Je sphere, $\mu(N)$, in the range $N=20-58$, with positions of the kinks labeled. (d) $E_{\mathrm{b}}(N)$ for $\mathrm{C}$ and $\mathrm{C}_{2}$ on a Je sphere of $R$ $=4.5 \AA$. Horizontal grid lines mark the literature values ${ }^{20}$ for $\mathrm{C}_{2}$ on various metal surfaces; those for $\mathrm{C}$ monomer in different locations, as labeled, are given as horizontal levels.

whose ionic potential in atomic units is 22

$$
V(r)= \begin{cases}-\frac{N}{r}, & r \geqslant R, \\ -\frac{N}{2 R}\left[3-\left(\frac{r}{R}\right)^{2}\right], & r \leqslant R,\end{cases}
$$

and energy $\frac{3}{5} N^{2} / R$ (code for generating Je spherical potential is provided in the supplementary material). The binding energy of $\mathrm{C}$ and $\mathrm{C}_{2}$ to the Je sphere is calculated as $E_{\mathrm{b}}=E(\mathrm{Je})+E(\mathrm{C})$ $-E(\mathrm{C} / \mathrm{Je})$, where $E(\cdot)$ is the total energy of the corresponding (relaxed) system. The behavior of this quantity separately as a function of $r_{s}$ and $N$ is plotted in Figs. 1(a) and 1(b). A comparison of $E_{\mathrm{b}}\left(r_{s}\right)$ and $E_{\mathrm{b}}(N)$, the latter displaying the same trend for $\mathrm{C}$ and $\mathrm{C}_{2}$ regardless of $R$, shows that $N$ is a more suitable variable and it will be used in the following. Minima in $E_{\mathrm{b}}$ correlate reasonably well [Fig. 1(b)] with the well-known electronic magic numbers associated with the closed electron shells in the spectrum of spherical Je. ${ }^{22,23}$ As a signature of the latter, the total magnetic moment $\mu(N)$ of the bare Je sphere, calculated for each $N$ in a narrower range [Fig. 1(c)], indeed, vanishes at $N=20,34,40,58$.

A detailed calculation of $E_{\mathrm{b}}$ over the same range of $N$ is given in Fig. 1(d), where we also provide available values from atomistic calculations ${ }^{20}$ for the same species in different configurations and host metals/surfaces. The binding energy of a $\mathrm{C}$ monomer on a $R=4.5 \AA \mathrm{Je}$ sphere, for instance, falls in the range of that for $\mathrm{C}$ on various $\mathrm{Ag}$ surface sites, or in bulk $\mathrm{Au}$ and $\mathrm{Ag}$, but only over a narrow range of $N \gtrsim 40$. Binding of $\mathrm{C}_{2} / \mathrm{Je}$, on the other hand, is well within that on a number of metals, except $\mathrm{Ni}\left(E_{\mathrm{b}}=6.8 \mathrm{eV}\right)$ and $\mathrm{Ag}\left(E_{\mathrm{b}}=5.8 \mathrm{eV}\right)$, which set the bounds of binding strength in fully atomistic calculations.

The above basic tests suggest that a Je sphere may serve as a rigid model of the catalyst-particle, whose properties as a fixed-volume electron reservoir are tunable via $N$ (or $r_{s}$ ). We further assess the model behavior in the case of Je in contact with ideal achiral CNTs, which is another prerequisite toward Je utility for exploring the growth of chiral CNTs. For a comprehensive comparison with accurate DFT treatment, Figs. 2(a) and 2(b) present the binding energies and interface energies $\gamma$ of zigzag and armchair tubes on metal clusters and surfaces/steps, respectively, as compiled from Ref. 20. Generally, coinage metals have lower binding energies compared to the late-transition metals. Among them, Ag has the lowest binding energies, Fig. 2(a), which originates from its lowest energy position for $d$ bands, ${ }^{24}$ and thereby the highest CNT/Ag interface energies $\gamma$, as shown in Fig. 2(b). Furthermore, we calculate $E_{\mathrm{b}}$ and $\gamma$ of CNT fragments on five Je variants of $R$ $=4.5 \AA$ and $N=34,42,47,48,49$. This choice is based on the fact that these values $\mu(N)$ vary from zero to a maximum, representing the cases of closed and open electronic shells, corresponding to minimum and maximum "open valence" of the clusters, Fig. 1(c). Calculations are performed for four achiral nanotubes, $(5,5),(6,6),(10,0)$, and $(11,0)$, and the results are summarized in Fig. 2(c). The binding energies for zigzag tubes are notably higher than those for armchair tubes (by $\approx 1 \mathrm{eV} /$ atom). The general trend is consistent with direct

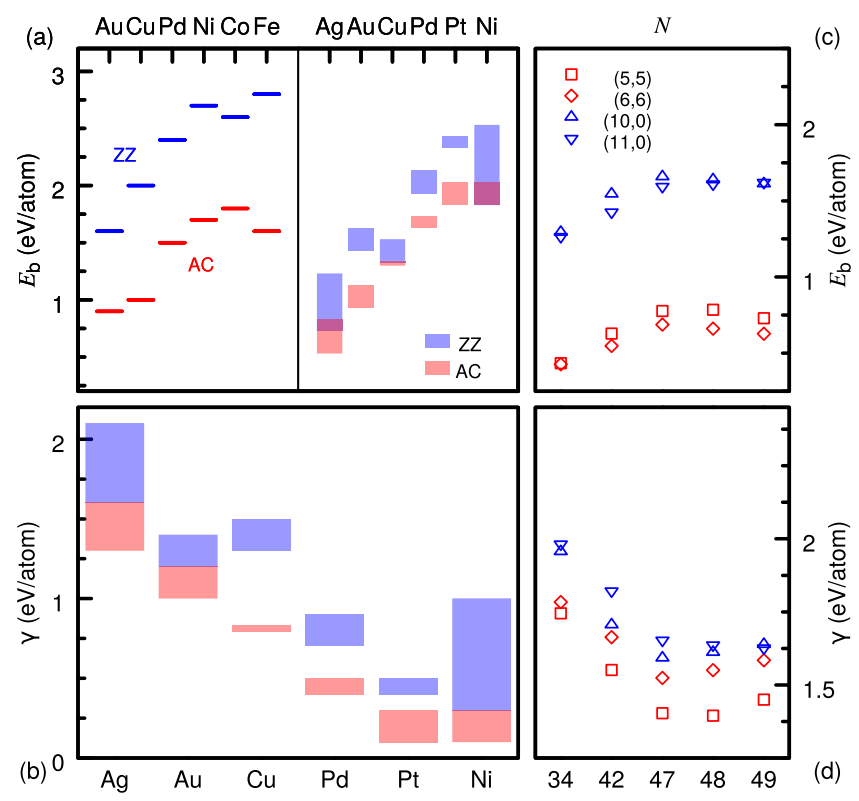

FIG. 2. (a) Binding energies of CNT to metal cluster (left) and various surfaces (right); (b) interface energies of zigzag and armchair CNTs on metal clusters and surfaces/steps (data from Refs. 20 and 25). The binding energies (c) and interface energies (d) calculated for $(5,5),(6,6),(10,0)$, and $(11,0)$ tubes on Je of $R=4.5 \AA$ for selected $N$. 
DFT calculations for single-walled CNTs on metal clusters. ${ }^{25}$ Moreover, by comparing the binding and interface energies on Je models [Figs. 2(c) and 2(d)] with those from fully atomistic DFT [Figs. 2(a) and 2(b)], the Je sphere would be reminiscent of Ag particles, as already indicated by the energetics of carbon monomer and dimer adsorption, Fig. 1(d), or more generally, as a group-Ia metal and, to some extent, group-Ib metals such as $\mathrm{Cu}$ and Ag. ${ }^{22}$

As noted earlier, the fixed-size Je sphere can be considered as an idealized, rigid/solid particle. In this case, we have recently demonstrated ${ }^{6}$ that the relative abundance in the growth product of single-walled CNTs of specific chiral angle $\chi$ and diameter $d, A(\chi, d)$, can exhibit a non-trivial, sharply peaked behavior at near-zero argument, $A \sim x \mathrm{e}^{-x}$, explaining the long-standing, puzzling preference for near-armchair $(n$, $n-1$ ) tubes in numerous experiments, ${ }^{8-10,26}$ in which case $x \equiv 30^{\circ}-\chi$. This behavior is understood as convolution of interface energetics ${ }^{3}\left(\sim \mathrm{e}^{-x}\right)$ and kinetics, ${ }^{5}$ viz., growth rate $(\sim x)$. If a catalyst, however, favors energetically a zigzag nanotube interface, then selectivity is expected for near-zigzag tubes, $(n, 1)$, with $x \equiv \chi / \sqrt{3}$. Following Ref. 6 , we compute separately the two major factors determining the CNT abundance $A(\chi, d) \equiv \mathcal{N}(\chi, d) \mathcal{R}(\chi, d)$ : the nucleation probability $\mathcal{N}$ and growth rate $\mathcal{R}$ for a tube of certain $\chi$ and $d$. The former factor requires the evaluation of the interface energy $\gamma(\chi)$ which is performed for seven selected chiral CNT types $(n, m)$ on Je spheres of three different sizes but the same $r_{s}$, as shown in Fig. 3(a). Different Je spheres have the same $r_{s}$ and their radii are chosen so as to result in a zero magnetic moment: $2 R=9$ $\AA, 10.8 \AA$, and $11.4 \AA$ corresponding to $N=34,58$, and 68 . The interface energy is calculated as $\pi d \gamma(\chi)=E_{\mathrm{b}}-E_{\text {edge }}$, where $E_{\text {edge }}$ is the energy of the open-edge (unpassivated) CNT of the same chirality $\chi$, and fitted to the analytical form, ${ }^{3}$

$$
\gamma=\gamma_{0} \cos (\chi+C)+\delta \cdot 4 \sin \chi \sin \left(30^{\circ}-\chi\right) / \cos \left(30^{\circ}+\chi\right)
$$

where $\delta$ is an armchair-zigzag-mix correction and $C$ is a chemical phase shift. ${ }^{3}$ This expression corresponds to a solid-like catalyst case.

The interface energy $\gamma(\chi)$ displays a familiar qualitative behavior with the two limits (zigzag and armchair) being local minima, Fig. 3(a). The Je spheres, however, favor the zigzag edge, by $\sim 0.1 \mathrm{eV} / \AA$ — a trend we have found earlier in atomistic calculations of CNTs with $d \simeq 8 \AA$ in contact with icosahedral $\mathrm{Fe}_{55}$ and rhombic dodecahedral bcc-Fe ${ }_{65}$ clusters. ${ }^{27}$ In contrast, for similar-diameter CNTs on a flat $\mathrm{Ni}(111)$ surface, ${ }^{6}$ the opposite case is realized, $\gamma_{\mathrm{A}}<\gamma_{\mathrm{Z}}$.

The calculation of the $\mathcal{R}(\chi, d)$ factor is again based on the protocol from Ref. 6: $(n, n)$ armchair tubes grow by addition of $\mathrm{C}_{2}$ to the open edge, and one computes the $n$ sequential steps, extending the CNT by a new "layer" of hexagons. For $(n, 0)$ zigzag tubes, however, the Klein edge ${ }^{28}$ is not stable on Je, forming a pentagon upon relaxation. We therefore consider a first step in which $3 \mathrm{C}$ atoms form the first hexagon, and adding dimers afterwards. The energy profiles of the "growth" process for three achiral tubes are shown in Fig. 3(b). The barrier for extending the corresponding CNT by a row of hexagons is then determined by the maximum-energy step
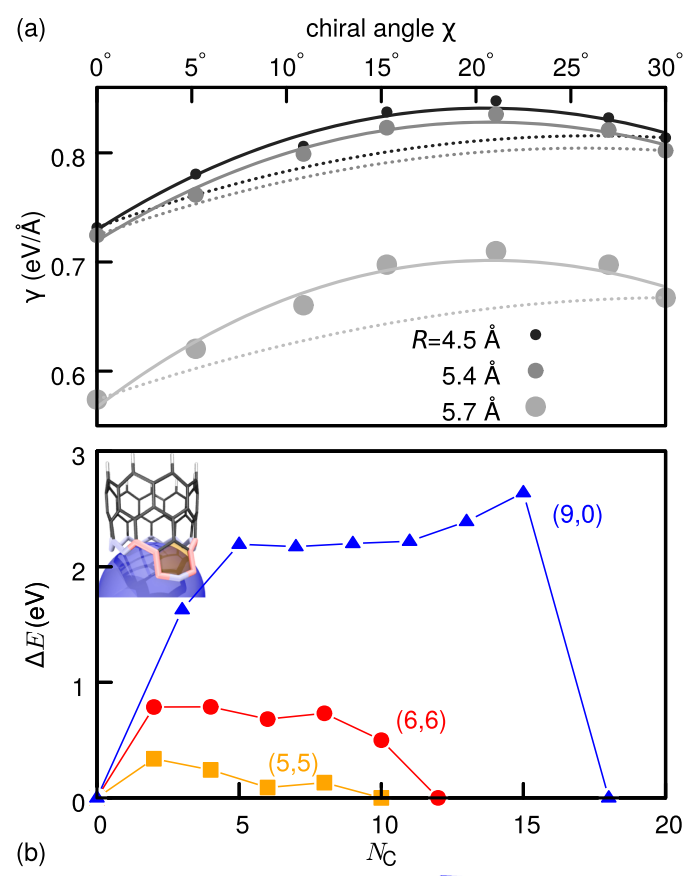

(b)

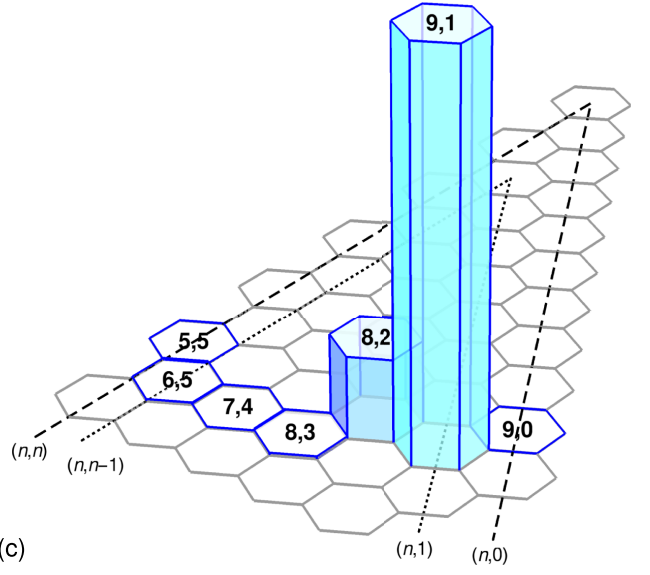

FIG. 3. (a) Interface energies for selected CNTs. The three Je spheres have the same $r_{s}$ but different $N$. The solid lines are fitted with analytical expression corresponding to solid-like catalyst case, Eq. (2), and the dotted lines corresponding to liquid-like catalyst case, ${ }^{3} \gamma(x)=2 \gamma_{A} \sin \chi+2 \gamma_{Z} \sin \left(30^{\circ}-\chi\right)$. (b) Free-energy profile during the nucleation and growth of a new ring of hexagons on achiral tube edges as a function of the number of carbon atoms added $N_{\mathrm{C}}$, for Je of $R=4.5 \AA$ A. (c) Computed CNT type distribution, resulting from (a) and (b).

along the carbon addition cycle. Qualitatively, $\Delta E$ matches to some extent the same quantity obtained from fully atomistic calculations ${ }^{6}$ (an initial steeper rise, and another slight increase before completing the new row of hexagons); however, these features are much less pronounced, especially for the armchair tubes.

Based on the above results, we estimate the CNT type distribution $A(\chi, d)$ on a spherical Je catalyst, as shown in Fig. 3(c), using the same provisions regarding the choice of temperature, $T=2700 \mathrm{~K}$, as in Ref. 6. Clearly, the Je sphere represents a case of a catalyst model that favors energetically a contact with a zigzag-edge CNT, leading to a sharp selectivity for the near-zigzag $(9,1)$ CNT. Such preferences, for instance, have been observed very recently for larger diameter tubes, achieved in temperature-modulated CVD growth using Fe catalyst. ${ }^{29}$ 
Departures from the ideal edge-growth scenario, where the perfect (curved) honeycomb lattice of the CNT wall is preserved, are also fundamentally important. The topological defects, a pentagon or a heptagon, can be limiting factors in the CNT growth process. For example, a pentagon shrinks the tube and, if not "healed," will result eventually in tube closure. The formation of a heptagon increases the CNT diameter and can cause edge detachment from the metal particle. As a further test for the model Je catalysts, we assess the difference in energy for creating separately each of these defects, $\varepsilon \equiv E_{7}-E_{5}$, at the chiral-CNT interface with a smaller and a larger Je spheres, Fig. 4. For all $(n, m)$ considered, a heptagon formation is energetically more costly, $\varepsilon>0$, regardless of Je radius, Fig. 4(a). More clear trends can be revealed when the energy difference is projected onto the tube/catalyst-diameter ratio $\xi \equiv d / 2 R$, Fig. 4(b). Linear fits to the two calculated $\varepsilon(\xi)$ sets indicate that there exists optimal $\xi_{0} \sim 0.1$ for given $R$, at which the energetics is balanced, $\varepsilon\left(\xi_{0}\right)=0$. The region ("Goldilocks zone") around $\xi_{0}$ may be interpreted as a growthsustaining feedback mechanism regarding defects formation at the CNT/Je growth front. For example, if a pentagon is energetically favored over a heptagon, $\varepsilon>0$, then the tube diameter $d$ decreases, reducing also $\varepsilon$, so that next a heptagon formation becomes more likely. A heptagon, as noted earlier, would increase $d$, avoiding the tube closure and thereby sustaining growth.

As a last example, we consider the formation of a pentagon-heptagon pair (517; at the interface with Je), which does not carry a disclination charge, but can change the CNT helicity type $(n, m))^{30,31}$ This is illustrated in the inset in Fig. 5 for the example of a 517 defect (essentially an edge dislocation) in a $(5,4)$ tube where the six possible orientations of its Burgers vector are indicated by arrows: $:^{32} \mathbf{b}= \pm(1,0), \pm(0,1)$, and $\pm(1,-1)$. The energy change $\Delta E$ upon the formation of 517 relative to the reference perfect $(n, m)$ edge is computed for three minimally chiral tubes and is shown as a function of relative diameter change $d / d_{0}$ in Fig. 5(a) and chiral angle change $\Delta \chi$ in Fig. 5(b). Both "projections" of $\Delta E$ reveal that only $(n, m) \rightarrow(n, m-1)$ changes have small $\Delta E<0.5 \mathrm{eV}$ and correspond to reduction of diameter and shift towards zigzag type, $\Delta \chi<0$. Formation of 517 in $(9,1)$ [along with those $\mathbf{b}$ in the

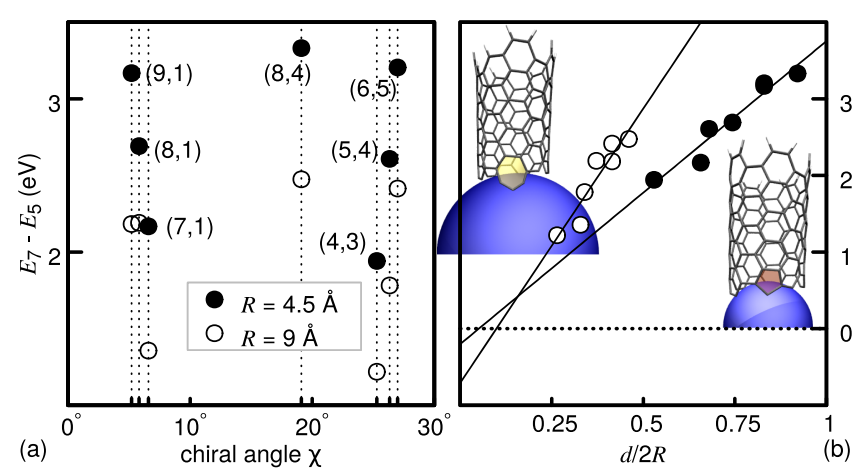

FIG. 4. (a) Heptagon-pentagon energy difference for three $(n, 1),(n, n-1)$, and one ( $2 m, m)$-type CNTs on Je of $R=4.5 \AA$ and $9 \AA$. (b) The same difference as a function of the tube/Je diameter ratio. Lines are fits to data sets on the same-diameter Je spheres. The insets illustrate a sample configuration of a heptagon on the larger- $R$ Je (left) and a pentagon on the smaller- $R$ Je (right) for the $(6,5)$ tube.
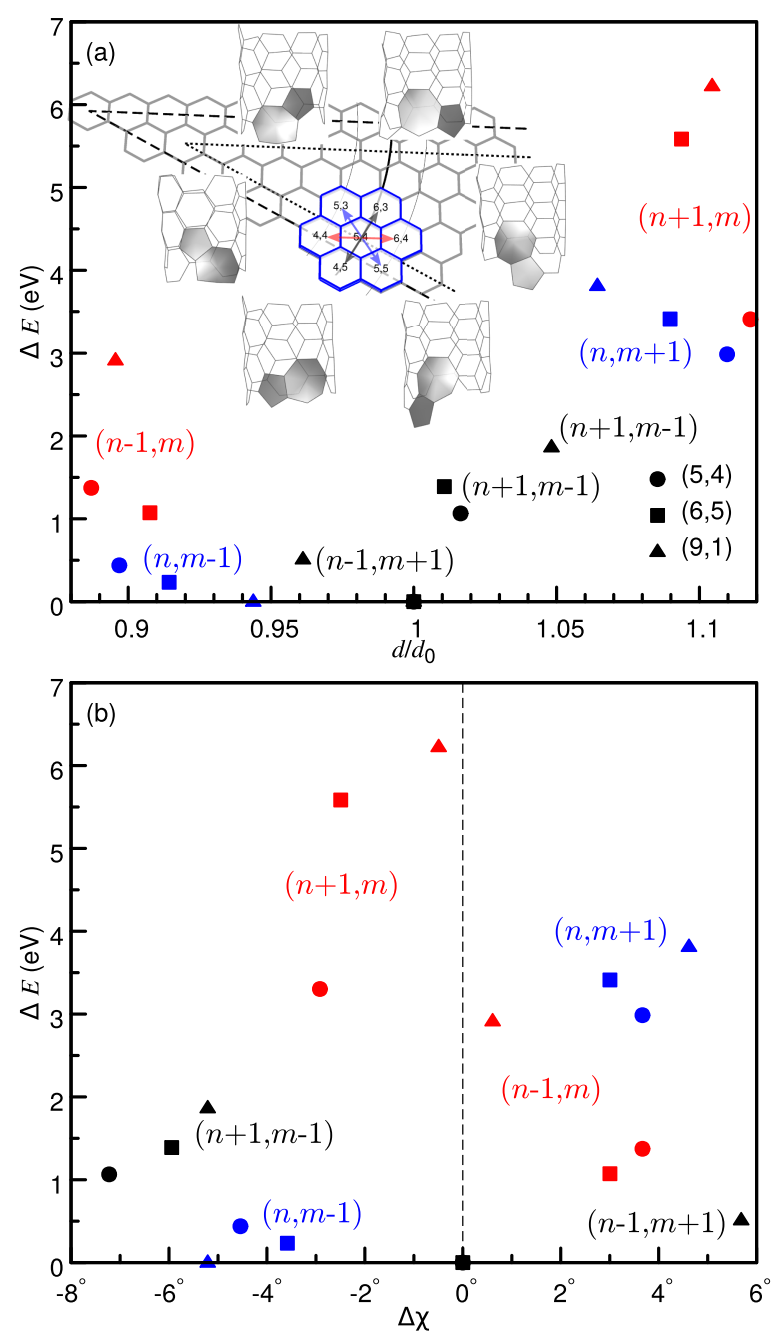

FIG. 5. (a) Energy cost $\Delta E$ versus relative diameter change $d / d_{0}$ due to an interface 517 defect for selected minimally chiral CNTs on Je of $R=4.5 \AA$. Note that a $(n-1, m+1)$-change starting from a near-armchair tube results in an enantiomer and therefore corresponding points for $(5,4)$ and $(6,5)$ coincide with the reference states; inset: an example of possible type changes of the $(5,4)$ tube. Arrows indicate $n \pm 1$ (red), $m \pm 1$ (blue), and $(n \pm 1, m \mp 1)$ (black) changes. (b) $\Delta E$ as a function of the corresponding chiral angle change $\Delta \chi \equiv \chi-\chi_{0}$.

near-armchair tubes, leading to chiral enantiomers, Fig. 5(a)] does not incur energy cost. Note also that the $(9,1) \rightarrow(9,0)$ transformation is associated with the largest reduction in the interface energy $\gamma$, Fig. 3(a), and appearance of a large kinetic barrier, Fig. 3(b).

In summary, the proposed spherical Je model appears to be an expedient surrogate metal catalyst that allows for a DFT-level treatment of the CNT/catalyst system at a considerably reduced computational effort (Je has been also used as annealing environment for carbon clusters in a semiempirical scheme ${ }^{33}$ ). Replacing an icosahedral $\mathrm{Ni}_{55}$ cluster, for instance, with $N=550$, by Je with the larger $N$ considered here reduces the number of electrons by an order of magnitude, as well as the number of degrees of freedom in which geometry optimization is to be performed. The softer Je potential, Eq. (1), should allow also for a lower plain-wave cutoff.

It is worth noting that the goal in such replacement here is to some extent opposite to the conventional Je as a model 
of alkali metal clusters, ${ }^{22}$ for example. While in the latter case the attempts have been to partially recover the eliminated ionic degrees of freedom, we have aimed at a structureless catalyst with less as possible specificity that can serve as a featureless electron reservoir. Figure 1 however clearly shows electronicshell fingerprints on the energetics of carbon species on the Je sphere. Since the electronic degrees of freedom are explicitly included, a finite electronic temperature $T$ will, however, smear the electronic shell effects for larger sizes, viz., $N$. For an order of magnitude estimate, one can use the single-particle energy levels spacing in a spherical potential, ${ }^{23,34,35} \sim \varepsilon_{\mathrm{F}} N^{-1 / 3}$, where $\varepsilon_{\mathrm{F}} \sim r_{s}^{-2}$ is the Fermi energy of the free electron gas. Thus, shell effect reduction may be expected for $\varepsilon_{\mathrm{F}} N^{-1 / 3} \lesssim T$, or Je sphere sizes $R \gtrsim N^{1 / 6} T^{-1 / 2}$ which, e.g., for $N \sim 100$ and $T \sim 0.1 \mathrm{eV}$ (typical growth temperatures), gives roughly $R \gtrsim 15 \AA$.

Simple variations of the proposed model are also possible (polyhedral Je, or a slab and stepped-slab; see Fig. S1 in the supplementary material) and make it a potentially useful tool for modeling catalytic growth of a broader set of systems and geometries.

\section{SUPPLEMENTARY MATERIAL}

See supplementary material for listing of Je spherical potential generator and some aspects of Je-slab implementations.

\section{ACKNOWLEDGMENTS}

This work was supported by the Office of Naval Research Grant No. N00014-15-1-2251. We thank Timur Galeev for his assistance in the early stages of the model benchmarking and validation. Computer resources were provided by the DAVinCI cluster at Rice University, acquired with funds from NSF Grant No. OCI-0959097.

\footnotetext{
${ }^{1}$ M. Davenport, Chem. Eng. News 93, 10 (2015).

${ }^{2}$ S. Noda, H. Sugime, K. Hasegawa, K. Kakehi, and Y. Shiratori, Jpn. J. Appl. Phys., Part 1 49, 02BA02 (2010).

${ }^{3}$ Y. Liu, A. Dobrinsky, and B. I. Yakobson, Phys. Rev. Lett. 105, 235502 (2010).

${ }^{4}$ Y. Magnin, A. Zappelli, H. Amara, F. Ducastelle, and C. Bichara, Phys. Rev. Lett. 115, 205502 (2015).

${ }^{5}$ F. Ding, A. R. Harutyunyan, and B. I. Yakobson, Proc. Natl. Acad. Sci. U. S. A. 106, 2506 (2009).
}

${ }^{6}$ V. I. Artyukhov, E. S. Penev, and B. I. Yakobson, Nat. Commun. 5, 4892 (2014).

${ }^{7}$ S. M. Bachilo, L. Balzano, J. E. Herrera, F. Pompeo, D. E. Resasco, and R. B. Weisman, J. Am. Chem. Soc. 125, 11186 (2003).

${ }^{8}$ G. Lolli, L. Zhang, L. Balzano, N. Sakulchaicharoen, Y. Tan, and D. E. Resasco, J. Phys. Chem. B 110, 2108 (2006).

${ }^{9}$ M. S. He, H. Jiang, B. L. Liu, P. V. Fedotov, A. I. Chernov, E. D. Obraztsova, F. Cavalca, J. B. Wagner, T. W. Hansen, I. V. Anoshkin, E. A. Obraztsova, A. V. Belkin, E. Sairanen, A. G. Nasibulin, J. Lehtonen, and E. I. Kauppinen, Sci. Rep. 3, 1460 (2013).

${ }^{10}$ H. Wang, L. Wei, F. Ren, Q. Wang, L. D. Pfefferle, G. L. Haller, and Y. Chen, ACS Nano 7, 614 (2013).

${ }^{11}$ F. Yang, X. Wang, D. Q. Zhang, J. Yang, D. Luo, Z. W. Xu, J. K. Wei, J. Q. Wang, Z. Xu, F. Peng, X. M. Li, R. M. Li, Y. L. Li, M. H. Li, X. D. Bai, F. Ding, and Y. Li, Nature 510, 522 (2014).

${ }^{12}$ J. R. Sanchez-Valencia, T. Dienel, O. Groning, I. Shorubalko, A. Mueller, M. Jansen, K. Amsharov, P. Ruffieux, and R. Fasel, Nature 512, 61 (2014).

${ }^{13}$ Y. Shibuta and S. Maruyama, Physica B 323, 187 (2002).

${ }^{14}$ M. A. Ribas, F. Ding, P. B. Balbuena, and B. I. Yakobson, J. Chem. Phys. 131, 224501 (2009).

${ }^{15}$ H. Amara, C. Bichara, and F. Ducastelle, Phys. Rev. Lett. 100, 056105 (2008).

${ }^{16}$ A. J. Page, Y. Ohta, S. Irle, and K. Morokuma, Acc. Chem. Res. 43, 1375 (2010).

${ }^{17}$ J. Y. Raty, F. Gygi, and G. Galli, Phys. Rev. Lett. 95, 096103 (2005).

${ }^{18}$ W. Ekardt, Phys. Rev. B 29, 1558 (1984).

${ }^{19}$ D. E. Beck, Solid State Commun. 49, 381 (1984).

${ }^{20}$ O. V. Yazyev and A. Pasquarello, Phys. Rev. Lett. 100, 156102 (2008).

${ }^{21}$ P. Giannozzi, S. Baroni, N. Bonini, M. Calandra, R. Car, C. Cavazzoni, D. Ceresoli, G. L. Chiarotti, M. Cococcioni, I. Dabo, A. Dal Corso, S. de Gironcoli, S. Fabris, G. Fratesi, R. Gebauer, U. Gerstmann, C. Gougoussis, A. Kokalj, M. Lazzeri, L. Martin-Samos, N. Marzari, F. Mauri, R. Mazzarello, S. Paolini, A. Pasquarello, L. Paulatto, C. Sbraccia, S. Scandolo, G. Sclauzero, A. P. Seitsonen, A. Smogunov, P. Umari, and R. M. Wentzcovitch, J. Phys.: Condens. Matter 21, 395502 (2009).

${ }^{22}$ M. Brack, Rev. Mod. Phys. 65, 677 (1993).

${ }^{23}$ W. A. de Heer, Rev. Mod. Phys. 65, 611 (1993).

${ }^{24}$ B. Hammer, Y. Morikawa, and J. K. Nørskov, Phys. Rev. Lett. 76, 2141 (1996).

${ }^{25}$ F. Ding, P. Larsson, J. A. Larsson, R. Ahuja, H. Duan, A. Rosén, and K. Bolton, Nano Lett. 8, 463 (2008).

${ }^{26}$ S. M. Bachilo, M. S. Strano, C. Kittrell, R. H. Hauge, R. E. Smalley, and R. B. Weisman, Science 298, 2361 (2002).

${ }^{27}$ E. S. Penev, V. I. Artyukhov, and B. I. Yakobson, ACS Nano 8, 1899 (2014).

${ }^{28}$ D. J. Klein, Chem. Phys. Lett. 217, 261 (1994).

${ }^{29}$ Q. Zhao, Z. Xu, Y. Hu, F. Ding, and J. Zhang, Sci. Adv. 2, e1501729 (2016).

${ }^{30}$ B. I. Dunlap, Phys. Rev. B 46, 1933 (1992).

${ }^{31}$ B. I. Dunlap, Phys. Rev. B 49, 5643 (1994).

${ }^{32}$ B. I. Yakobson, Appl. Phys. Lett. 72, 918 (1998).

${ }^{33}$ I. Chaudhuri, M. Yu, C. S. Jayanthi, and S. Y. Wu, J. Phys.: Condens. Matter 26, 115301 (2014).

${ }^{34}$ K. L. Clemenger, "Spheroidal shell structure and static electric polarizabilities of alkali metal clusters," Ph.D. thesis, University of California, Berkeley, 1985, Sec. 2.4.

${ }^{35}$ H. Nishioka, K. Hansen, and B. R. Mottelson, Phys. Rev. B 42, 9377 (1990). 